\title{
Cosmic-ray interactions with the Sun
}

\section{N. Mazziotta, ${ }^{a, *}$ P. De La Torre Luque, ${ }^{a, b, c}$ L. Di Venere, ${ }^{a, b}$ A. Fassò,${ }^{d}$ A. Ferrari, ${ }^{e, g}$ F. Loparco, ${ }^{a, b}$ P. R. Sala ${ }^{e}$ and D. Serini ${ }^{a}$}

${ }^{a}$ Istituto Nazionale di Fisica Nucleare, Sezione di Bari, via Orabona 4, I-70126 Bari, Italy

${ }^{b}$ Dipartimento di Fisica “M. Merlin”, dell'Università e del Politecnico di Bari via Amendola 173, I-70126 Bari, Italy

${ }^{c}$ Currently at The Oskar Klein Centre, Department of Physics, Stockholm University AlbaNova SE-10691 Stockholm, Sweden

d 13 Passage Hamo, CH-1262 EYSINS, Switzerland

e Istituto Nazionale di Fisica Nucleare, Sezione di Milano

Via Celoria,16, 20133 Milano, Italy

${ }^{g}$ Institute for Astroparticle Physics (IAP), Karlsruhe Institute of Technology (KIT)

P.O. Box 3640, Karlsruhe, Germany

E-mail: mazziotta@ba.infn.it

The solar disk is a bright gamma-ray source in the sky. The interactions of cosmic rays with the solar atmosphere produce secondary particles which can reach the Earth. In this work we present a comprehensive calculation of the yields of secondary particles such as gamma-rays, electrons, positrons, neutrons and neutrinos, performed with the FLUKA code. We also estimate the intensity at the Sun and the fluxes at the Earth of these secondary particles by folding their yields with the intensities of cosmic rays impinging on the solar surface. The results are sensitive to the assumptions on the magnetic field near the Sun and to the cosmic-ray transport in the magnetic field in the inner solar system.

$37^{\text {th }}$ International Cosmic Ray Conference (ICRC 2021)

July 12th-23rd, 2021

Online - Berlin, Germany

\footnotetext{
*Presenter
} 


\section{Introduction}

Cosmic rays reaching the Sun and the planets in the Solar system can produce secondary particles, such as gamma rays and neutrinos, due to their interactions with the surfaces or the atmospheres of the bodies. The Moon [1,2] and the Earth [3] are bright sources of gamma rays, both well visible with space-borne instruments. Lunar and terrestrial emissions are mainly due to the hadronic interactions of cosmic-ray nuclei with the lunar surface and with the upper layers of the Earth's atmosphere respectively.

The Sun is also a bright source of high-energy gamma rays, and its emission consists of two components: the first one, called disk emission, is due to cosmic-ray nuclei interacting with the solar surface [4, 5], and is localized around the solar disk; the second one, which is due to the inverse Compton scatterings of cosmic-ray electrons (and positrons) with the solar optical photons, is a diffuse component and extends up to tens of degrees from the Sun [4, 6-8].

In this work we have performed a full simulation with the FLUKA code [9-11] to calculate the yields of secondary particles produced by the interactions of cosmic rays with the Sun (disk emission). Full details on this work are given in ref. [12].

\section{Simulation set-up}

The FLUKA code has been used to simulate the propagation and the interactions of cosmic rays with the solar atmosphere. We use a reference frame in which the Sun is described as a sphere of radius $R_{\odot}=6.9551 \times 10^{10} \mathrm{~cm}$, with its rotation axis along the $z$-axis. The radial profiles of the chemical composition, density, temperature and pressure of the Sun are included in the geometry. We have also implemented several models of the magnetic field in the region close to the Sun (inner magnetic field), while for the interplanetary magnetic field (outer magnetic field) we have used the Parker model.

To evaluate the yields of secondary particles from the Sun we have simulated several samples of protons, electrons and ${ }^{4} \mathrm{He}$ nuclei with different kinetic energies impinging a sphere of radius $R_{S S}=2.5 R_{\odot}$ surrounding the Sun, with an isotropic and uniform flux. The generation sphere corresponds to the boundary between the inner and outer magnetic field regions. We have simulated primary particles in a wide energy range from $0.1 \mathrm{GeV} / \mathrm{n}$ to $100 \mathrm{TeV} / \mathrm{n}$, while the energies of secondary particles have been simulated down to $100 \mathrm{keV}$.

The chemical composition profile of the Sun is derived from the Standard Solar Models (SSMs) for the interior of the Sun, provided by Ref. [13] ${ }^{1}$. The main components are $\mathrm{H}$ and ${ }^{4} \mathrm{He}$, while the abundances of heavier isotopes are below $1 \%$. We have extrapolated this model outside the Sun, assuming that the chemical composition of the atmosphere is the same as that at $r=R_{\odot}$, since most of cosmic-ray interactions will take place in the solar atmosphere close to the surface of the Sun.

We use the model provided by Ref. [14] to define the radial profiles of density, temperature and pressure. The mode extends up to about $500 \mathrm{~km}$ above the solar surface and we have extrapolated it to higher altitudes, up to about $1400 \mathrm{~km}$. In the FLUKA simulation set-up we have implemented 100 layers (i.e. shells) with different densities and chemical compositions, divided in three sets equally spaced on a logarithmic density scale: the external 40 layers from about $10^{-13} \mathrm{~g} / \mathrm{cm}^{3}$ up

\footnotetext{
${ }^{1}$ Data file http://www.ice.csic.es/personal/aldos/Solar_Data_files/struct_b16_gs98.dat
} 
to $10^{-3} \mathrm{~g} / \mathrm{cm}^{3}$, the middle 40 layers from $10^{-3} \mathrm{~g} / \mathrm{cm}^{3}$ to $10^{-1} \mathrm{~g} / \mathrm{cm}^{3}$ and the inner 20 layers with density $>10^{-1} \mathrm{~g} / \mathrm{cm}^{3}$.

The magnetic field near the Sun is complex and strongly time-dependent. A widely adopted model is the potential field source surface (PFSS) model $[15,16]$, in which the field is purely radial on a sphere of radius $R_{S S}=2.5 R_{\odot}$ (source surface). We have implemented in our simulation the field maps taken from the Solar Dynamics Observatory Joint Science Operations Center (JSOC) [17], which are calculated starting photospheric magnetic field observations. The field maps are available for each Carrington Rotation number (CRn), starting from CRn 2097 (May-June 2010).

The interplanetary magnetic field (IMF) affects the cosmic-ray propagation in the solar system. In our simulation we describe the IMF using the Parker model [18] for $r>R_{S S}$. The values of the tilt angle $\alpha$ and of its polarity are taken from the Wilcox Solar Observatory public website ${ }^{2}$, while the intensity of the magnetic field at the Earth $B_{0}$ and the velocity of the solar wind $v_{S W}$ are taken from the observations of the ACE satellite extracted from the NASA/GSFC's OMNI dataset [19].

\section{Results}

The differential intensity of secondary particles of the species $s$ emitted from the Sun (at the production) is given by:

$$
I_{S}\left(E_{S}\right)=\sum_{i} \int Y_{s, i}\left(E_{S} \mid E_{k}\right) I_{i}\left(E_{k}\right) d E_{k}
$$

where $I_{i}\left(E_{k}\right)$ is the intensity of the $i$-th species of cosmic-ray primaries at the Sun and $Y_{s, i}\left(E_{s} \mid E_{k}\right)$ is the yield of secondary particles $s$ produced from the $i$-th species of cosmic-ray primaries (here $i=p, e^{-}$and ${ }^{4} \mathrm{He}$ ), which is evaluated by counting the secondary particles which escape from the generation surface.

The flux of secondaries observed by a detector at Earth is given by:

$$
\phi_{S}\left(E_{s}\right)=\frac{\pi R_{S S}^{2}}{R_{E}^{2}} I_{S}\left(E_{S}\right) \mathcal{F}\left(E_{S}\right)
$$

where $\mathcal{F}\left(E_{s}\right)$ is the fraction of secondaries with energy $E_{s}$ which are able to reach the Earth's orbit from the Sun. In our simulation we assume that the Earth's orbit lays on a sphere centered on the Sun with radius $R_{E}=1 \mathrm{AU}$. We point out here that not all secondaries emitted outwards from the Sun are able to reach the Earth.

Charged particles are deflected by the IMF and, depending on their energy and initial direction, can be sent back to the Sun without reaching the Earth's orbit. In addition, there are some species of unstable secondaries, such as neutrons, which can decay during their journey from the Sun to the Earth. In these cases, the fraction of secondaries reaching the Earth will be $\mathcal{F}\left(E_{s}\right) \leq 1$. On the other hand, for the secondary gamma rays and neutrinos we assume $\mathcal{F}\left(E_{S}\right)=1$.

Cosmic rays impinging on the solar atmosphere are those which can reach the Sun from the interplanetary space. Hence the intensities $I_{i}\left(E_{k}\right)$ of the various cosmic-ray primaries in eq. 1 are those at the surface of the generation sphere of radius $R_{S S}$, which differ from those measured at Earth, since not all cosmic rays reaching the Earth are able to continue their journey to the Sun.

\footnotetext{
${ }^{2}$ http://wso.stanford.edu/Tilts.html
} 


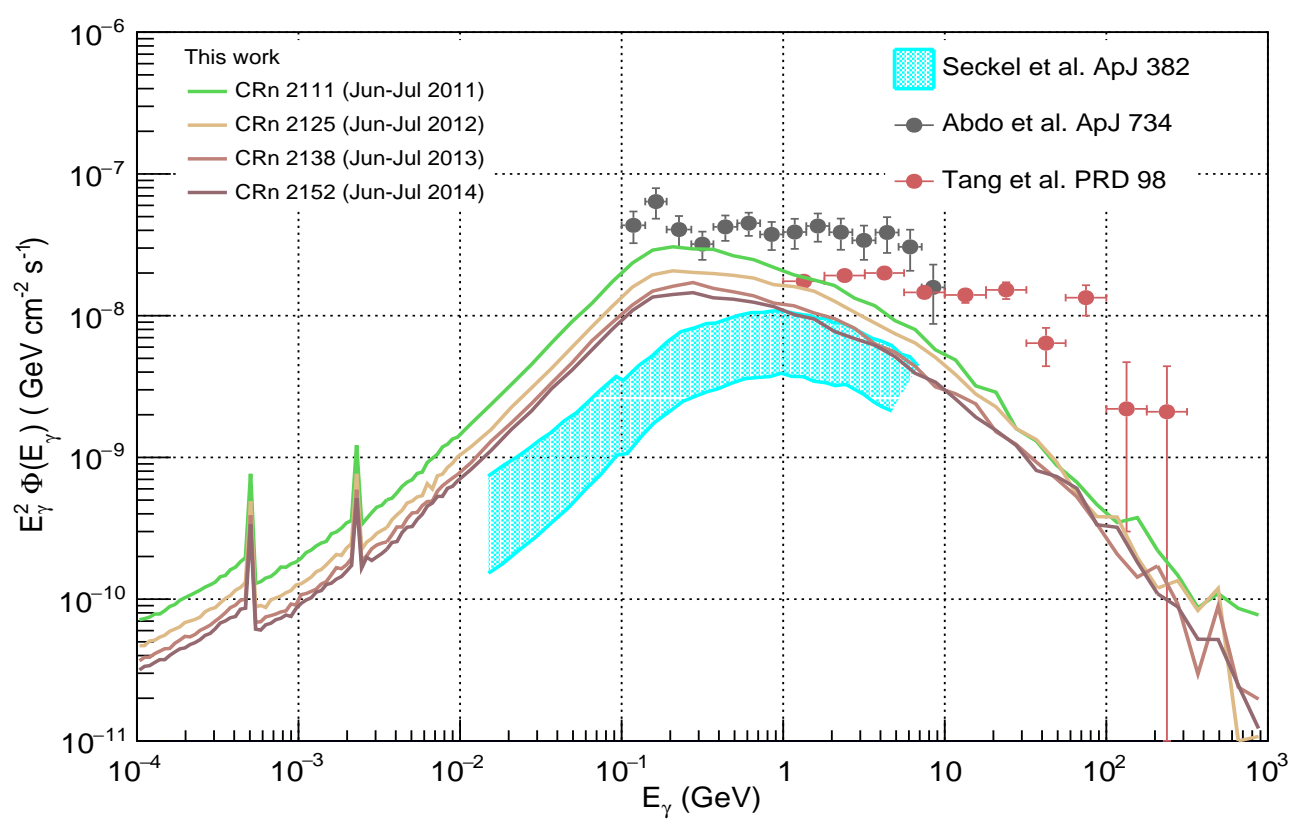

Figure 1: Gamma-ray fluxes at the Earth for four different PFSS inner magnetic field Carrington Rotation number. From top to bottom: CRn 2111, 2125, 2138 and 2152, respectively.

To evaluate the cosmic-ray intensities at the Sun we have used the custom version of the code HelioProp $[20,21]^{3}$, which describes the transport of cosmic rays in the solar system. We have simulated sets of pseudo-particles injected on the surface of the generation sphere with an isotropic and uniform distribution.

We use the cosmic-ray intensities at Earth measured by AMS-02: the proton intensity is taken from Ref. [22], the helium intensity is taken from Refs. [23, 24] and the electron ${ }^{4}$ intensity is taken from Ref. [26]. We also use the AMS-02 spectra measured for different Bartels'rotations (BR) $[25,27]$.

Figure 1 shows the gamma-ray fluxes at the Earth evaluated with our simulation set-up for four different CRn (2111, 2125, 2138 and 2152) spanning the period from June 2011 to June 2014, that covers the AMS-02 measurements. The calculated gamma-ray fluxes are slightly different at low energies $(<1 \mathrm{GeV})$, due to the effect of the heliospheric magnetic field that affects both the cosmic-ray intensity at the Sun and the secondary yields.

The secondary emissivity of the Sun is strongly dependent on the intensity of the magnetic field close to the solar surface. To study this effect, we have selected the epoch corresponding to CRn 2111 and we have implemented three additional magnetic field configurations:

1. $B=0$, i.e. the inner magnetic field is switched off;

2. $0.1 \times$ PFSS, i.e. the original PFSS magnetic field intensity is reduced of a factor 10 ;

${ }^{3}$ See also https://github. com/cosmicrays.

${ }^{4} \mathrm{We}$ use the total intensity of electrons and positrons, and we refer to them as electrons. 


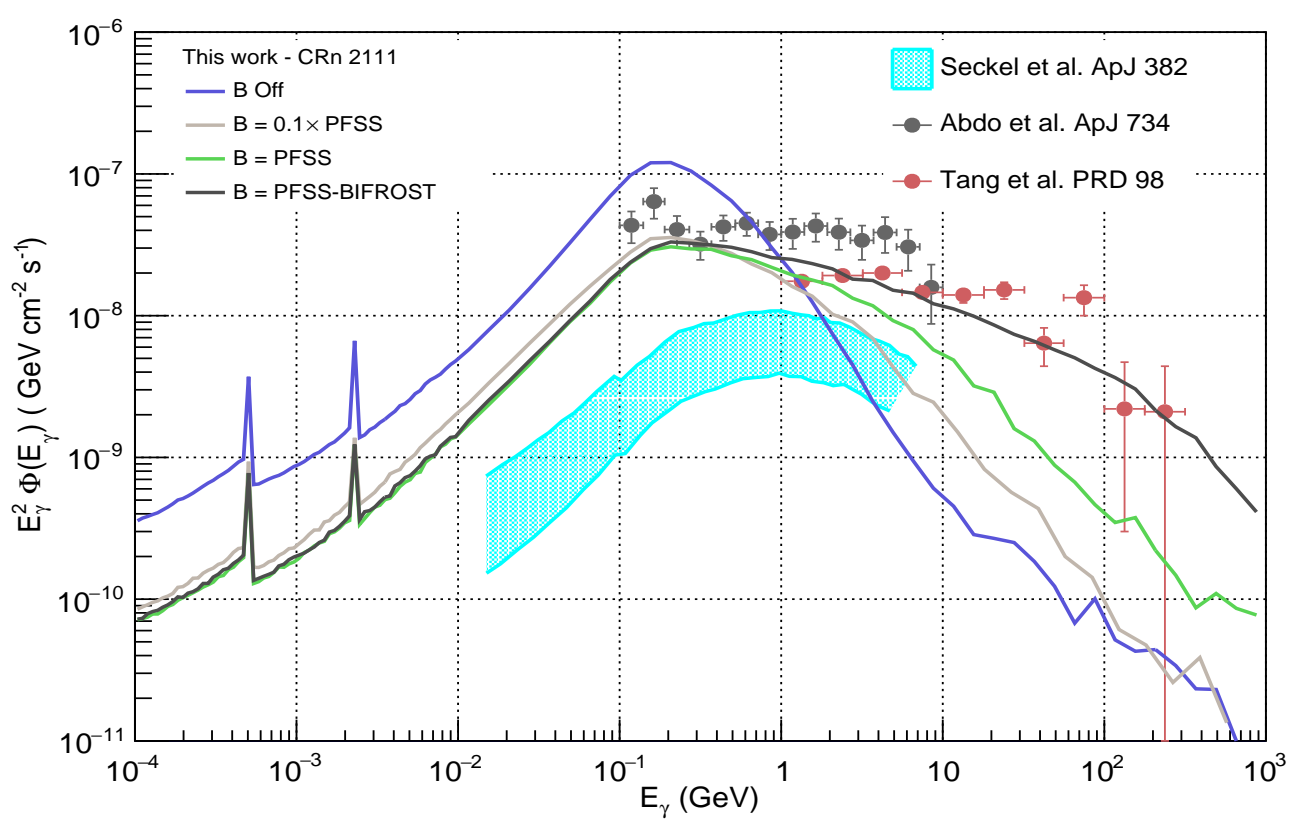

Figure 2: Gamma-ray fluxes at the Earth for four different magnetic field configurations: no inner $B$ field; $B$ field intenisty reduced by a factor 10 with respect to the nominal PFSS configuration; nominal PFSS configuration and enhanced magnetic field according to the BIFROST model.

3. enhanced $B$ field configuration near the Sun $\left(r / R_{\odot}<1.01\right)$ following the BIFROST model [2830], i.e. the original PFSS maps near the Sun are enhanced to follow the BIFROST profile ${ }^{5}$.

Figure 2 shows the gamma-ray fluxes at the Earth with the four different configurations for the inner magnetic field, i.e. the nominal model and the three alternative models illustrated above.

The gamma-ray flux without inner magnetic field is significantly enhanced at low energies with respect to the flux in presence of magnetic field, while for gamma-ray energies above $10 \mathrm{GeV}$ the flux increases as the magnetic field increases. If the solar magnetic field is suppressed, lowenergy cosmic rays can reach the solar surface, inducing hadronic showers with secondary particles propagating in the outer space. The presence of a solar magnetic field reduces the probability that low-energy cosmic rays can reach the Sun, but increases the interaction probability of high-energy cosmic rays, since they move along curved trajectories in a strong and non-uniform magnetic field and the path length increases as the magnetic field increases. This effect is well visible when comparing the gamma-ray flux with $B=0$ with the one with enhanced $B$ field configuration.

In Figs. 1 and 2 we also show the experimental results obtained with the Fermi LAT [31] data for the disk component. The two LAT data sets correspond to a period of 1.5 years from August 2008 to January 2010, between the end of the $23^{r d}$ and the beginning of the $24^{\text {th }}$ solar cycle [4], and to a period of 9 years from August 2008 to July 2017, spanning an almost full 11-years solar cycle [32]. We remark here that these measurements have been performed in different time

\footnotetext{
${ }^{5}$ The BIFROST simulation is available for a limited region of the Sun. The enhancement factor is about 25 at the solar surface.
} 
windows from those covered by our simulation. However, from Fig. 1 we see that the data seem to indicate that the measured spectral energy distribution is almost flat up to beyond $10 \mathrm{GeV}$, while our simulation predicts a peak in the spectral energy distribution of gamma rays at energies around $200 \mathrm{MeV}$. Nonetheless, from Fig. 2 we see that the peak becomes broader as the inner magnetic field intensity increases.

In Figs. 1 and 2 we also show the predictions of the gamma-ray flux at the Earth made by Seckel et al. [5] under their nominal assumptions, taken from figure 7 in Ref. [5]). The expected gamma-ray flux in each CRn considered in the present work is always larger than the flux predicted in Ref. [5].

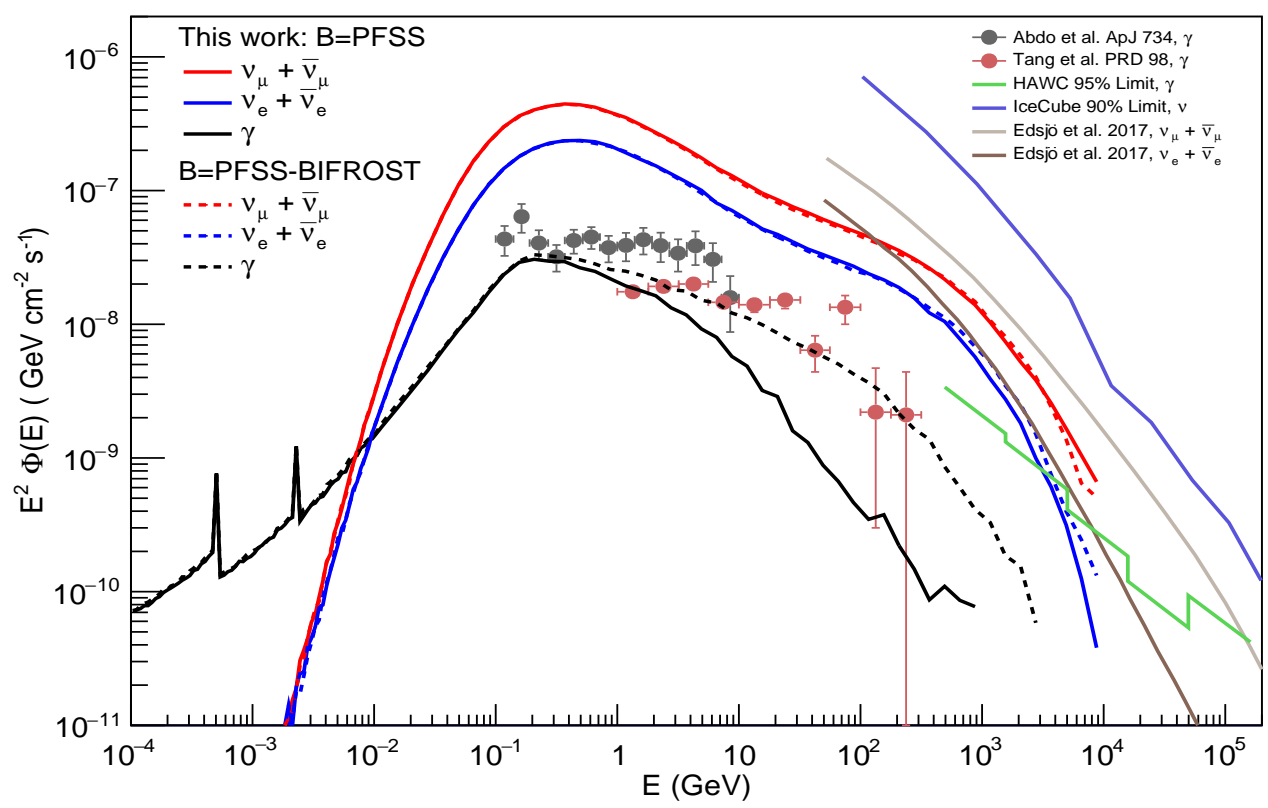

Figure 3: Gamma-ray and neutrino fluxes at the Earth calculated for the CRn 2111 with two models for the Sun magnetic field: PFSS (continuous line) and enhanced model according to the BIFROST prediction (dashed line). Black line: gamma-ray; red line: $v_{\mu}+\bar{v}_{\mu}$; blue line: $v_{e}+\bar{v}_{e}$. The prediction of neutrinos by Ref. [35] is also shown. In addition, we include results from gamma-ray observations from Fermi-LAT [4, 32], gamma-ray HAWC's 95\% limit [36] and neutrinos IceCube's 90\% limit [37].

In Fig. 3 we show the predictions of our simulation of the fluxes at Earth of gamma rays and neutrinos. The simulations have been performed with the standard PFSS (CRn 2111) solar magnetic field and with the enhanced one according to the BIFROST profile.

\section{Conclusions}

We have implemented a full simulation with the FLUKA code to calculate the yields of secondary particles produced by the interactions of primary cosmic rays with the solar atmosphere. Our simulation includes the current state-of-art models and data available to describe the solar atmosphere, the magnetic field nearby the Sun and in the interplanetary space. 
We have studied the effect of the magnetic field on the secondary yields by implementing different models for the inner magnetic field. We found that the yields are strong affected by the intensity of the magnetic field, even in the high-energy region of the emission in the outer space. Recent developments of numerical solutions of a magnetohydrodynamical (MHD) model together with the current observation of the Parker Solar Probe [33, 34] could provide new insights to get a more realistic description of the magnetic field nearby the Sun.

The calculated solar gamma-ray flux at the Earth has been compared with the measurements performed using the Fermi-LAT data. The gamma-ray flux from the solar disk above $1 \mathrm{GeV}$ exhibits a harder spectrum $\left(\sim E_{\gamma}^{-2.2}\right)$ than that of cosmic rays $\left(\sim E_{p, H e}^{-2.7}\right)$. This behaviour would require a high-intensity magnetic field configuration nearby the Sun, up to a factor 20 larger than the field predicted by the PFSS model used in the present simulation. The magnetic field should also affect the inverse Compton gamma-ray emission close to the Sun, since also cosmic-ray electrons and positrons move along curved trajectories, whose lengths determine the interaction probability with solar optical photons. To get a full picture of the solar gamma-ray emission, the inverse Compton scattering needs to be calculated in presence of strong and irregular magnetic field.

\section{Acknowledgments}

We acknowledge the FLUKA collaboration for providing and supporting the code; the Joint Science Operations Center (JSOC) data; the NASA/GSFC's Space Physics Data Facility's OMNIWeb (or CDAWeb or ftp) service, and OMNI data; the Community Coordinated Modeling Center at Goddard Space Flight Center through their public Runs on Request system (http : //ccmc . gsfc . nasa. gov); the Hinode Science Data Centre Europe (http://sdc.uio.no/search/simulations). This work has been realized using the RECAS computing infrastructure in Bari (https://www . recas-bari.it/index.php/en/).

\section{References}

[1] A. A. Abdo et al. [Fermi-LAT] Astrophys. J. 758, no.2, 140 (2012)

[2] M. Ackermann et al. [Fermi-LAT], Phys. Rev. D 93, no.8, 082001 (2016) [arXiv:1604.03349].

[3] A. A. Abdo et al. [Fermi-LAT], Phys. Rev. D 80, 122004 (2009) [arXiv:0912.1868].

[4] A. A. Abdo et al. [Fermi-LAT], Astrophys. J. 734, 116 (2011) [arXiv:1104.2093]

[5] D. Seckel, T. Stanev and T. K. Gaisser, Astrophys. J. 382, 652-666 (1991)

[6] E. Orlando and A. W. Strong, Astron. Astrophys. 480, 847 (2008) [arXiv:0801.2178]

[7] E. Orlando and A. Strong, Astrophys. Space Sci. 309, 359 (2007) [arXiv:astro-ph/0607563]

[8] I. V. Moskalenko, T. A. Porter and S. W. Digel, Astrophys. J. Lett. 652, L65-L68 (2006) [erratum: Astrophys. J. Lett. 664, L143 (2007)] [arXiv:astro-ph/0607521].

[9] A. Ferrari, P. R. Sala, A. Fasso and J. Ranft, http://cds . cern. ch/record/898301 
[10] T. T. Böhlen et al. Nucl. Data Sheets 120, 211-214 (2014)

[11] G. Battistoni et al. Annals Nucl. Energy 82, 10-18 (2015)

[12] M. N. Mazziotta et al. Phys. Rev. D 101, no.8, 083011 (2020) [arXiv:2001.09933]

[13] N. Vinyoles et al. Astrophys. J. 835, no.2, 202 (2017) [arXiv:1611.09867]

[14] J. Christensen-Dalsgaard et al. Science 272, 1286-1292 (1996)

[15] Schatten, K. H., Wilcox, J. M., \& Ness, N. F. 1969, Solar Physics, 6, 442

[16] Hakamada, K. 1995, Solar Physics, 159, 89. doi:10.1007/BF00733033

[17] http://jsoc.stanford.edu/ajax/lookdata.html?ds=hmi .pfss_synop P.H. Scherrer et al. Solar Phys 162, 129-188 (1995)

[18] E. N. Parker, Astrophys. J. 128, 664-676 (1958)

[19] https://omniweb.sci.gsfc.nasa.gov/ftpbrowser/ace_merge.html J. H. King and N. E. Papitashvili, Journal of Geophysical Research, 110, A02104

[20] L. Maccione, Phys. Rev. Lett. 110, no.8, 081101 (2013) [arXiv:1211.6905]

[21] A. Vittino, C. Evoli and D. Gaggero, PoS ICRC2017, 024 (2018) [arXiv:1707.09003]

[22] M. Aguilar et al. [AMS], Phys. Rev. Lett. 114, 171103 (2015)

[23] M. Aguilar et al. [AMS], Phys. Rev. Lett. 115, no.21, 211101 (2015)

[24] M. Aguilar et al. [AMS], Phys. Rev. Lett. 119, no.25, 251101 (2017)

[25] M. Aguilar et al. [AMS], Phys. Rev. Lett. 121, no.5, 051101 (2018)

[26] M. Aguilar et al. [AMS], Phys. Rev. Lett. 113, 221102 (2014)

[27] M. Aguilar et al. [AMS], Phys. Rev. Lett. 121, no.5, 051102 (2018)

[28] M. Carlsson et al. Astronomy \& Astrophysics, 585 (2016) A4

[29] B. V. Gudiksen et al. Astron. Astrophys. 531, A154 (2011) [arXiv:1105.6306]

[30] http://sdc.uio.no/search/simulations?sim=en024048_hion

[31] W. B. Atwood et al. [Fermi-LAT], Astrophys. J. 697, 1071-1102 (2009) [arXiv:0902.1089]

[32] Q. W. Tang et al. Phys. Rev. D 98, no.6, 063019 (2018) [arXiv:1804.06846]

[33] Fox, N. J., Velli, M. C., Bale, S. D., et al. 2016, Space Science Reviews, 204, 7

[34] Riley, P., Downs, C., Linker, J. A., et al. 2019, The Astrophysical Journal Letters, 874, L15

[35] J. Edsjo, J. Elevant, R. Enberg and C. Niblaeus, JCAP 06, 033 (2017) [arXiv:1704.02892]

[36] A. Albert et al. [HAWC], Phys. Rev. D 98, no.12, 123011 (2018) [arXiv:1808.05620]

[37] M. G. Aartsen et al. [IceCube], JCAP 02 (2021) 025 [arXiv:1912.13135] 